01

\title{
Электронные спектры поглощения нейтральных и заряженных молекулярных кластеров серебра
}

\author{
() М.В. Столярчук, А.И. Сидоров \\ Университет ИТМО, \\ 197101 Санкт-Петербург, Россия \\ e-mail: maxim.stolyarchuk@corp.ifmo.ru
}

Поступила в редакцию 13.02.2018 г.

В окончательной редакции 16.05.2018 г.

\begin{abstract}
В рамках теории функционала плотности (DFT) промоделированы структурные, энергетические и оптические свойства заряженных и нейтральных молекулярных кластеров (МК) серебра $\mathrm{Ag}_{n}(n=2-5)$. Показано, что электронный спектр поглощения нейтральных МК смещен в сторону меньших энергий по сравнению с заряженными. Силы осцилляторов нейтральных МК в основном больше сил осцилляторов заряженных МК. Проведено сопоставление результатов моделирования с полученными ранее экспериментальными результатами для стекол с МК серебра.
\end{abstract}

DOI: $10.21883 /$ OS.2018.09.46540.42-18

\section{Введение}

Субнаноразмерные молекулярные кластеры (МК) по своим свойствам занимают промежуточное положение между отдельными атомами, наночастицами и объемным состоянием вещества. Так, наличие квантоворазмерных эффектов у МК металлов, состоящих из нескольких атомов, определяет их свойства, подобные молекулярным: дискретный спектр энергетических уровней, отсутствие плазмонного резонанса и др. [1]. Оптические свойства МК металлов являются предметом экспериментальных и теоретических исследований из-за возможности их практических применений в качестве материалов для солнечной энергетики, устройств фотоники и сенсорики [2-4]. Молекулярные кластеры, состоящие из атомов элементов 11 группы $(\mathrm{Cu}, \mathrm{Ag}, \mathrm{Au})$, диспергированные в различных средах, включая неорганические матрицы, обладают поглощением в видимой и УФ областях оптического спектра, а также флуоресцируют [5]. В отличие от макроскопического состояния вещества, где добавление или удаление нескольких электронов практически не вызывает изменений, в МК такое добавление или удаление оказывает существенное влияние на электронную структуру, что сказывается и на оптических свойствах.

На сегодняшний день существует значительное количество экспериментальных и теоретических работ, посвященных исследованию различных свойств изолированных МК серебра. Экспериментальные исследования МК серебра проводились в том числе с помощью фотоэлектронной спектроскопии [6] и оптической спектроскопии в цеолитах и твердых инертных газах [7-9]. Ряд теоретических работ был направлен на изучение равновесной (стабильной) геометрии основного состояния, энергетических и электронных свойств МК металлов [10-12]. В работе [13] методом нестационарного приближения локальной плотности (TDLDA) были изучены оптические свойства нейтральных МК. Позднее разными группами были представлены исследования нейтральных МК серебра с применением нестационарной теории функционала плотности с иными функционалами $[8,14]$. В то время как свойства нейтральных МК обширно изучены различными теоретическими методами, наблюдается дефицит работ по изучению строения и оптических свойств заряженных МК металлов, в частности, по сравнению их энергетических свойств со свойствами нейтральных МК. Так, в работе [15] с помощью метода, основанного на формализме уравнений движений (ЕОМ-CC), рассматриваются возбужденные состояния нейтральных $\mathrm{Ag}_{2-4}$ и положительно заряженных $\mathrm{Ag}_{2-4}^{+}$.

Ранее нами было экспериментально показано [16], что в фототерморефрактивных стеклах, содержащих серебро и фотосенсибилизатор - ионы $\mathrm{Ce}^{3+}$, серебро исходно находится в виде ионов и положительно заряженных МК. Такие стекла обладают чрезвычайно слабой люминесценцией в видимой области спектра при возбуждении УФ излучением. При воздействии на такие стекла интенсивного УФ излучения, попадающего в полосу поглощения ионов церия $(\lambda=305-310 \mathrm{~nm})$, происходит фотоионизация $\mathrm{Ce}^{3+}$ с образованием в стекле свободных электронов. Электроны захватываются ионами $\mathrm{Ag}^{+}$и заряженными $\mathrm{MK} \mathrm{Ag}_{n}^{q+}$, что переводит часть заряженных МК в нейтральное состояние. При этом происходит существенное изменение спектров люминесценции и возбуждения люминесценции (рис. 1) и значительное увеличение интенсивности люминесценции стекол с МК серебра в видимой области спектра (см. вставку в рис. $1, a)$. Из рис. $1, b$ видно, что спектры возбуждения люминесценции структурированы, что указывает на наличие в стекле нескольких люминесцентных центров. Как было показано в работе [16], вклад в люминесценцию в данном случае вносят в основном атомы $\mathrm{Ag}^{0}$ и нейтральные $\mathrm{MK} \mathrm{Ag}_{n}(n=2-4)$. Термообработка таких стекол при температуре, меньшей температуры стеклования, приводит к увеличению размера МК 

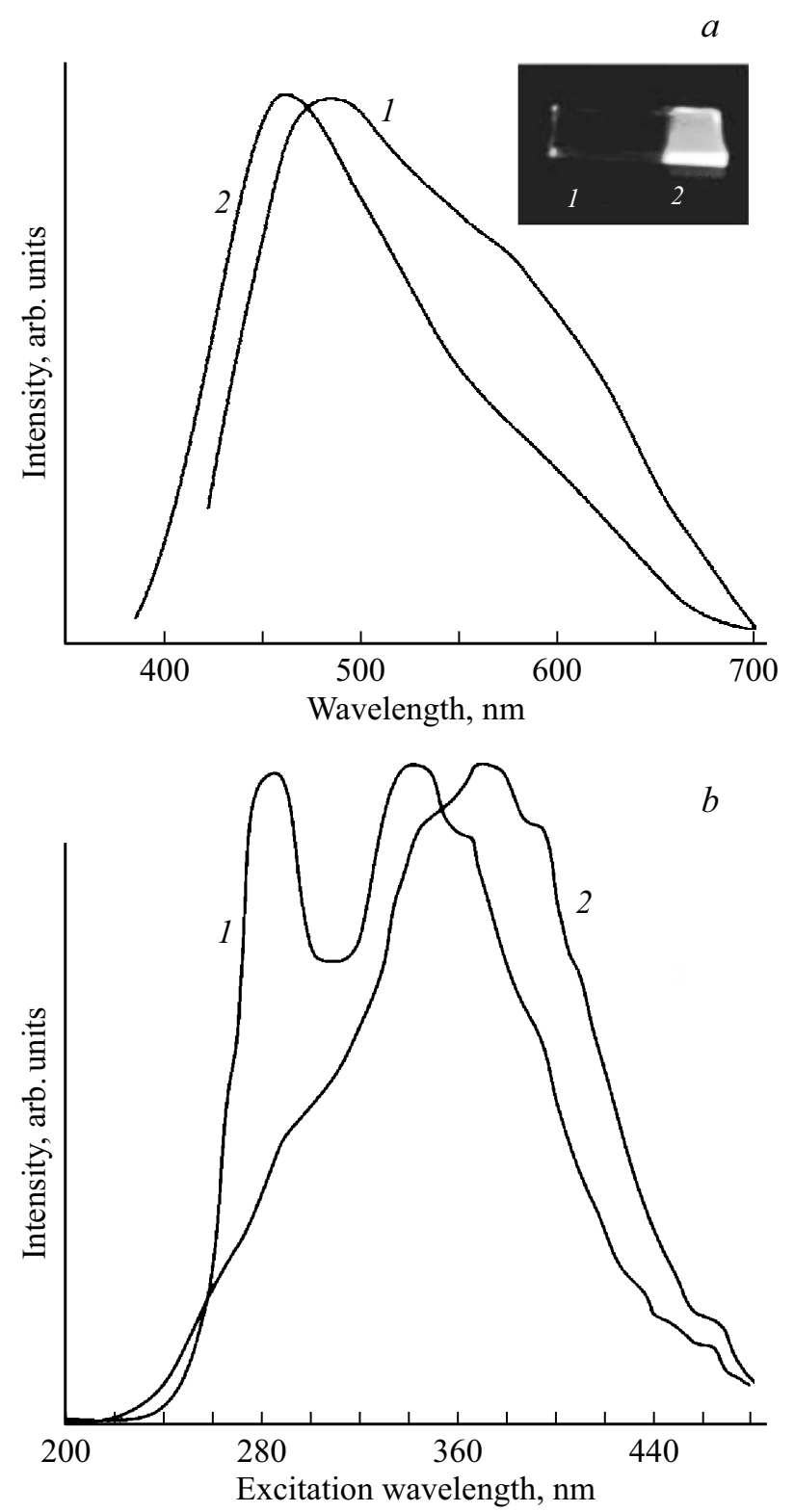

Рис. 1. Нормированные спектры люминесценции (a) и возбуждения люминесценции $(b)$ заряженных $(1)$ и нейтральных (2) МК серебра в фототерморефрактивном стекле. $a-$ длина волны возбуждения люминесценции $350 \mathrm{~nm} . b-$ длина волны люминесценции $570 \mathrm{~nm}$. На вставке - фотография люминесценции фототерморефрактивного стекла с областями, содержащими заряженные (1) и нейтральные (2) МК серебра. Длина волны возбуждения $365 \mathrm{~nm}$.

(увеличению $n$ ) и их концентрации за счет термической диффузии атомов серебра, что также влияет на спектры люминесценции и их интенсивность [17]. Таким образом, появляется возможность управлять спектральнолюминесцентными свойствами фототерморефрактивных стекол. В связи с этим возникает ряд вопросов, имеющих как научное, так и прикладное значение. В частности, как влияют зарядовое состояние и размеры МК серебра на их спектрально-люминесцентные свойства? Кроме того, представляет интерес выяснение того, какой вклад вносят в люминесценцию в видимой области спектра МК серебра с различным количеством атомов.

\section{Методика моделирования}

Геометрия и оптические свойства МК серебра исследовались в рамках теории функционала плотности (DFT) с применением программного пакета Amsterdam Density Functional (ADF2014.11) [18]. Уравнения КонаШэма решались с использованием градиентно-обобщенного обменно-корреляционного функционала РВE [19] вместе с трехэкспоненциальным базисным набором орбиталей слэтеровского типа с включением двух поляризационных функций (TZ2P). Молекулярные кластеры исследовались в вакуумном приближении. В то же время необходимо принимать во внимание, что в силикатных стеклах МК серебра располагаются вблизи структурных дефектов сетки стекла, что может оказывать влияние на их свойства, в частности приводить к уширению спектра, изменению амплитуды спектральных полос и их сдвигу по спектру. Все исследуемые структуры подвергались геометрической оптимизации с последующим колебательным анализом, целью которого было установление наличия мнимых частот. Отсутствие мнимых частот свидетельствует, что найденная стационарная точка отвечает минимуму потенциальной энергии. Системы с открытой электронной оболочкой рассматривались в рамках неограниченного по спину формализма. Расчет возбужденных состояний производился с использованием теории функционала плотности, зависящей от времени (TDDFT) [20] на уровне CAM-B3LYP/QZ4P. Как было показано в [21], ранжированный (range-separated) функционал CAM-B3LYP [22] аккуратнее описывает асимптотическое поведение обменно-корреляционного потенциала, что позволяет корректнее рассчитать вертикальный спектр электронных возбуждений МК переходных металлов.

\section{Результаты и обсуждение}

\section{Равновесные геометрии МК и их энергетические свойства}

Наиболее стабильные геометрические структуры после оптимизации приведены на рис. 2 вместе с длинами межатомных связей. Расчет проводился методом PBE/TZ2P. Полученные равновесные структуры находятся в хорошем согласии с результатами большинства предыдущих исследований $[11,12]$. Длина связи $\mathrm{Ag}_{2}$ составила $2.572 \AA$, что близко к экспериментальному значению $2.533 \AA$ [23]. Для $\mathrm{Ag}_{3}$ наблюдается эффект Яна-Теллера [24], вследствие чего МК является не равносторонним треугольником $\left(D_{3 h}\right)$, а представляет собой равнобедренный треугольник $\left(C_{2 v}\right)$. Для рассматриваемых нейтральных $\mathrm{MK} \mathrm{Ag}_{n}$ характерна плоская 
Таблица 1. Точечная группа симметрии Г, основное состояние, среднее межатомное расстояние $r_{e}(\AA)$, энергия связи в пересчете

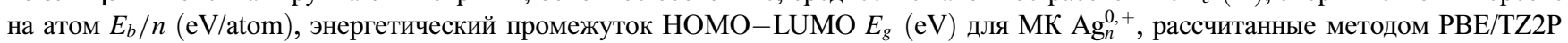

\begin{tabular}{c|c|c|c|c|c|c|c|c|c|c}
\hline \multirow{2}{*}{ МК } & \multicolumn{5}{|c|}{$\mathrm{Ag}_{n}$} & \multicolumn{5}{c}{$\mathrm{Ag}_{n}^{+}$} \\
\cline { 2 - 10 } & $\Gamma$ & Состояние & $r_{e}$ & $E_{b} / n$ & $E_{g}$ & $\Gamma$ & Состояние & $r_{e}$ & $E_{b} / n$ & $E_{g}$ \\
\hline $\mathrm{Ag}_{2}$ & $D_{\infty h}$ & ${ }^{1} \Sigma_{g}^{+}$ & 2.57 & 0.88 & 2.05 & $D_{\infty h}$ & ${ }^{2} \Sigma_{g}^{+}$ & 2.73 & 0.89 & 0.56 \\
$\mathrm{Ag}_{3}$ & $C_{2 v}$ & ${ }^{2} B_{2}$ & 2.76 & 0.87 & 0.28 & $D_{3 h}$ & ${ }^{1} A_{1}$ & 2.69 & 1.55 & 3.01 \\
$\mathrm{Ag}_{4}$ & $D_{2 h}$ & ${ }^{1} A_{g}$ & 2.74 & 1.15 & 0.84 & $D_{2 h}$ & ${ }^{2} B_{1 u}$ & 2.76 & 1.48 & 0.34 \\
$\mathrm{Ag}_{5}$ & $C_{2 v}$ & ${ }^{2} A_{1}$ & 2.72 & 1.25 & 0.36 & $D_{2 d}$ & ${ }^{1} A_{1}$ & 2.78 & 1.53 & 1.96
\end{tabular}

структура. Для катионных $\mathrm{MK} \mathrm{Ag}_{n}^{+}$переход между двумерной и трехмерной структурой наблюдается при $n=5$. В этом случае плоская трапецевидная структуpa $\mathrm{Ag}_{5}^{+}$с симметрией $C_{2 v}$ преобразуется в ромбовидную $\left(D_{2 d}\right)$. Из приведенных на рис. 2 данных видно, что с увеличением количества атомов длины связи $\mathrm{Ag}-\mathrm{Ag}$ нейтральных МК увеличиваются и приближаются к экспериментальному значению межатомного расстояния для массивного металлического серебра (2.89 А) [25]. Нейтральные МК, за исключением $\mathrm{Ag}_{3}$, образуют более компактную структуру по сравнению с заряженными (см. также табл. 1). Для оценки термодинамической стабильности нейтральных и заряженных МК была ис-

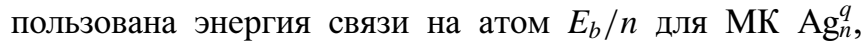
которая определяется как

$$
E_{b} / n=\left[(n-1) E(\mathrm{Ag})+E\left(\mathrm{Ag}^{q}\right)-E\left(\mathrm{Ag}_{n}^{q}\right)\right] / n,
$$

где $E\left(\mathrm{Ag}_{n}^{q}\right)$ - полная энергия $\mathrm{MK}, E(\mathrm{Ag})$ и $E\left(\mathrm{Ag}^{q}\right)-$ энергии отдельного атома и иона, $q=0,+1-$ заряд.

Рассчитанные значения $E_{b} / n$ представлены в табл. 1 . Энергии связи в пересчете на один атом для МК с четным числом атомов больше, чем соответствующие величины для случая нечетного числа атомов. Помимо этого, энергия связи $E_{b} / n$ постепенно возрастает с увеличением количества атомов в МК и приближается к когезивной энергии объемного серебра, которая равна $2.95 \mathrm{eV}$ [25]. Однако $\mathrm{Ag}_{4}^{+}$менее стабилен, чем $\mathrm{Ag}_{3}^{+}$, что свидетельствует о большей стабильности катионных МК с закрытой электронной оболочкой (с нечетным количеством атомов). Полученные данные показывают, что рассматриваемые катионные МК более стабильны по сравнению с нейтральными.

Количество валентных электронов в МК влияет на энергетическую стабильность за счет изменения электронной плотности. Энергетический промежуток $E_{g}$ отражает энергию, необходимую для переноса электрона с высшей занятой молекулярной орбитали (HOMO) на низшую вакантную молекулярную орбиталь (LUMO) и характеризует кинетическую стабильность системы. Как отмечалось ранее, системы с открытой оболочкой рассчитывались спин-поляризованным методом. Для систем с открытой электронной оболочкой $E_{g}$ относится к энергетическому зазору между $\alpha$-HOMO и $\beta$-LUMO.
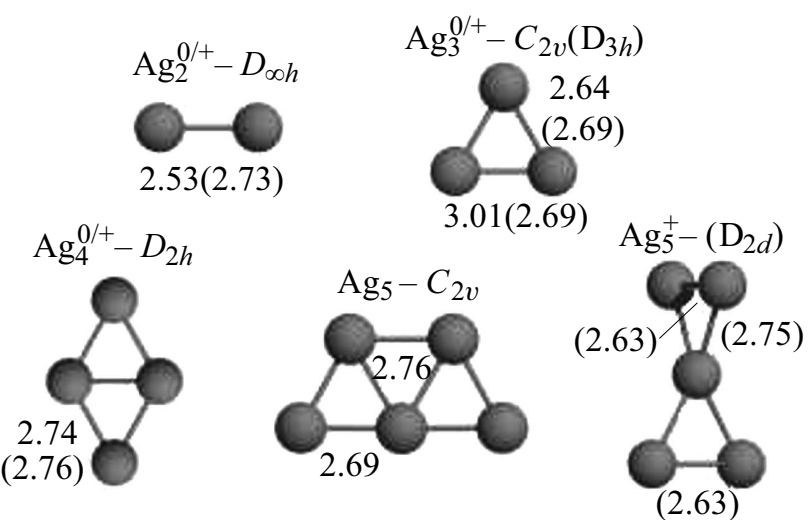

Рис. 2. Наиболее стабильные конфигурации $\mathrm{Ag}_{n}^{0,+}(n=2-5)$, рассчитанные методом PBE/TZ2Р. Длины связей приведены в ангстремах. В скобках указаны длины связей для заряженных МК.

Из табл. 1 видно, что значения энергетического зазора $E_{g}$ осциллируют с изменением размера МК. Максимумы наблюдаются для систем со спаренными электронами. При этом граничные орбитали нейтральных МК лежат выше по сравнению с соответствующими орбиталями катионных МК.

\section{Электронные спектры поглощения MK}

Оптический спектр поглощения может быть охарактеризован энергией электронных переходов и их силой осциллятора. При расчетах дискретный спектр уширялся с учетом распределения Лоренца с параметром полуширины $\gamma=60 \mathrm{meV}$. Электронные переходы были проанализированы в рамках занятых и виртуальных молекулярных орбиталей, которые участвуют в переходе между основным и возбужденным состояниями (табл. 2). На рис. 3 приведены спектры поглощения нейтральных и катионных МК в УФ и видимой областях спектра. Рассчитанные энергии перехода и силы осциллятора хорошо согласуются с известными экспериментальными данными для МК серебра в матрицах инертных газов [8] и результатами теоретических расчетов для нейтральных МК [22,24].

Спектр $\mathrm{Ag}_{2}$ характеризуется двумя основными пиками поглощения, за которые отвечают переходы с 

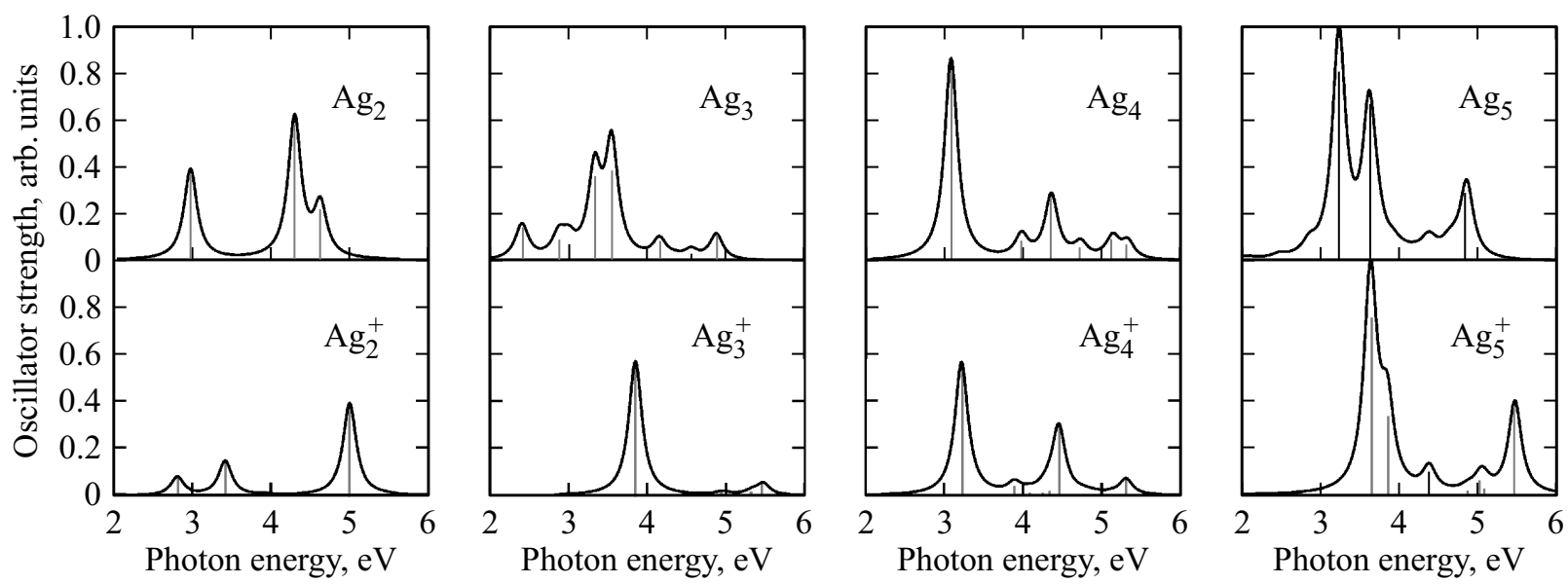

Pис. 3. Электронные спектры поглощения нейтральных и заряженных $\mathrm{MK} \mathrm{Ag}_{n}$, рассчитанные методом CAM-B3LYP/QZ4P.

энергией $3.09, \quad 5.21 \mathrm{eV}$ и силами осцилляторов 0.39 и 0.6 соответственно (рис. 3). По своей природе данные полосы поглощения описываются преимущественно вкладами электронных конфигураций HOMO $\left(10 \sigma_{g}^{+}\right) \rightarrow \operatorname{LUMO}\left(10 \sigma_{u}^{+}\right)(96 \%) \quad$ и $\quad \mathrm{HOMO} \rightarrow$ $\rightarrow$ LUMO-1 $\left(6 \pi_{u}\right)(87 \%)$ соответственно (табл. 2). Для других рассматриваемых МК в наиболее интенсивных переходах также принимают участие пограничные молекулярные орбитали. На рис. 4 показано положение энергий и силы осцилляторов переходов с максимальной интенсивностью в зависимости от количества атомов в МК. Наиболее интенсивный переход для $\mathrm{Ag}_{2}^{+}$смещен в коротковолновую область по сравнению с переходами для $\mathrm{Ag}_{2}$. Сила осциллятора наиболее интенсивного перехода для нейтральных МК, за исключением $\mathrm{Ag}_{3}$, больше, чем для заряженных. С увеличением размера МК положение наиболее интенсивного перехода смещается на $0.15-0.7 \mathrm{eV}$ в область меньших энергий. Для МК с нечетным количеством атомов сила осциллятора перехода с максимальной интенсивностью незначительно изменяется после удаления электрона. Данные результаты могут дать объяснение экспериментально полученным фактам, а именно увеличению интенсивности люминесценции в видимой области спектра и длинноволновому смещению спектров возбуждения при трансформации заряженных МК серебра в нейтральные. В настоящей работе подразумевалось, что интенсивность люминесценции пропорциональна силе осциллятора поглощения.

Представляет практический интерес на основании полученных результатов провести анализ, каков вклад в люминесценцию вносят различные МК для фиксированных длин волн возбуждения. Одним из перспективных применений люминесцентных стекол с МК серебра является спектральная даун-конверсия коротковолнового излучения в видимую область спектра в светодиодах белого свечения. Источниками УФ излучения в данном случае предполагается использовать излучающие полупроводниковые диоды с длинами волн излучения $365 \mathrm{~nm}$

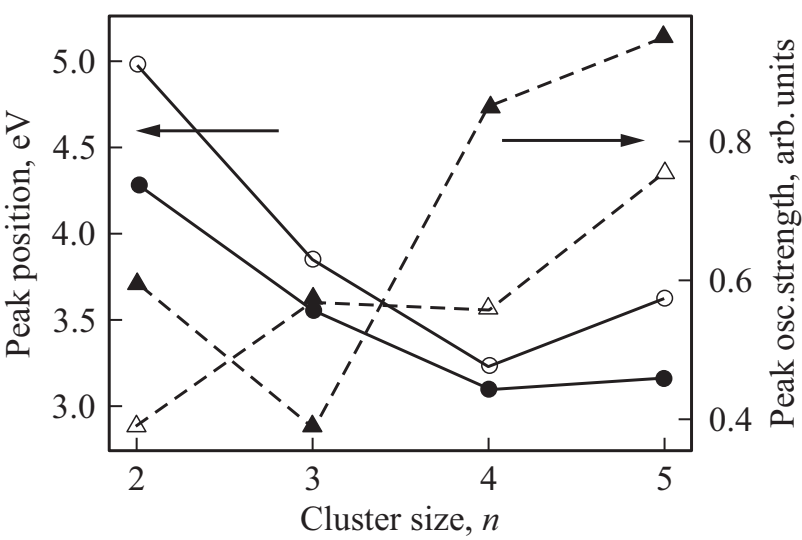

Рис. 4. Положение энергий и сил осцилляторов переходов с максимальной интенсивностью. Темные маркеры соответствуют нейтральным МК, светлые - заряженным.

$(E=3.39 \mathrm{eV})$ и $405 \mathrm{~nm}(E=3.06 \mathrm{eV})$. Такие диоды в настоящее время имеют мощность до нескольких ватт, они доступны и относительно дешевы. Вклад люминесценции ионов и атомов серебра, также присутствующих в стеклах, в данном случае не рассматривается, так как они имеют полосы возбуждения люминесценции в спектральном интервале $250-330 \mathrm{~nm}$. Из рис. 3,4 и табл. 2 следует, что для $\lambda=365 \mathrm{~nm}$ основной вклад в

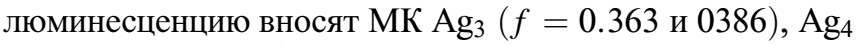
$(f=0.847)$ и $\mathrm{Ag}_{5}^{+}(f=0.697)$. Незначительный вклад могут вносить заряженные $\mathrm{MK} \mathrm{Ag}_{2}^{+}(f=0.145)$. Для длины волны $405 \mathrm{~nm}$ основной вклад в люминесценцию будут вносить МК $\mathrm{Ag}_{2}(f=0.385), \mathrm{Ag}_{4}(f=0.847)$, $\mathrm{Ag}_{4}^{+}(f=0.558)$ и $\mathrm{Ag}_{5}(f=0.947)$. Отсюда следует, что для эффективной даун-конверсии излучения в светодиодах белого свечения при синтезе люминесцентных стекол с МК серебра и дальнейшей их обработке необходимо создавать условия, при которых в стекле будет формироваться максимальное количество МК серебра, отмеченных выше. 
Таблица 2. Энергии $E$, длины волн $\lambda$ и силы осцилляторов $f$ наиболее интенсивных переходов, рассчитанные методом CAM-B3LYP/QZ4P, с указанием конфигураций в рамках молекулярных орбиталей

\begin{tabular}{|c|c|c|c|c|c|}
\hline Кластер & Состояние & $E, \mathrm{eV}$ & $\lambda, \mathrm{nm}$ & $f$ & Конфигурация перехода, \% \\
\hline $\mathrm{Ag}_{2}$ & $\begin{array}{l}1^{1} \Sigma_{u}^{+} \\
\mathbf{1}^{\mathbf{1}} \boldsymbol{\Pi}_{\mathbf{u}} \\
2^{1} \Pi_{u}\end{array}$ & $\begin{array}{l}3.09 \\
4.29 \\
4.62\end{array}$ & $\begin{array}{l}401 \\
\mathbf{2 8 9} \\
268\end{array}$ & $\begin{array}{l}0.385 \\
\mathbf{0 . 5 9 9} \\
0.108\end{array}$ & $\begin{array}{l}10 \sigma_{g}^{+} \rightarrow 10 \sigma_{u}^{+}, 96 \% \\
10 \sigma_{g}^{+} \rightarrow 6 \pi_{u}, 87 \% \\
5 \pi_{g} \rightarrow 10 \sigma_{u}^{+}, 86 \%\end{array}$ \\
\hline $\mathrm{Ag}_{2}^{+}$ & $\begin{array}{l}1^{2} \Sigma_{u}^{+} \\
2^{2} \Pi_{u} \\
2^{2} \Sigma_{u}\end{array}$ & $\begin{array}{l}2.82 \\
3.42 \\
4.99\end{array}$ & $\begin{array}{l}440 \\
362 \\
\mathbf{2 4 8}\end{array}$ & $\begin{array}{l}0.077 \\
0.145 \\
\mathbf{0 . 3 9 0}\end{array}$ & $\begin{array}{l}10 \alpha \sigma_{g}^{+} \rightarrow 10 \alpha \sigma_{u}^{+}, 56 \% \\
9 \beta \sigma_{u}^{+} \rightarrow 10 \beta \sigma_{g}^{+}, 42 \% \\
9 \beta \sigma_{u}^{+} \rightarrow 10 \beta \sigma_{g}^{+}, 54 \% \\
10 \alpha \sigma_{g}^{+} \rightarrow 10 \alpha \sigma_{u}^{+}, 42 \% \\
10 \alpha \sigma_{g}^{+} \rightarrow 6 \alpha \pi_{u}, 95 \%\end{array}$ \\
\hline $\mathrm{Ag}_{3}$ & $\begin{array}{l}2 B_{2} \\
4 B_{2}\end{array}$ & $\begin{array}{l}2.41 \\
3.34\end{array}$ & $\begin{array}{l}514 \\
371\end{array}$ & $\begin{array}{l}0.144 \\
0.363\end{array}$ & $\begin{array}{l}22 \alpha b_{2} \rightarrow 30 \alpha a_{1}, 72 \% \\
22 \alpha b_{2} \rightarrow 31 \alpha a_{1}, 47 \% \\
28 \alpha a_{1} \rightarrow 22 \alpha b_{2}, 40 \%\end{array}$ \\
\hline $\mathrm{Ag}_{3}^{+}$ & $\begin{array}{l}\mathbf{1} \mathbf{E}^{\prime} \\
2 A^{\prime \prime}\end{array}$ & $\begin{array}{l}3.85 \\
5.53\end{array}$ & $\begin{array}{l}322 \\
224\end{array}$ & $\begin{array}{l}\mathbf{0 . 5 6 8} \\
0.353\end{array}$ & $\begin{array}{l}12 a_{1}^{\prime} \rightarrow 17 e_{1}^{\prime}, 94 \% \\
12 a_{1}^{\prime} \rightarrow 6 a_{2}^{\prime \prime}, 88 \%\end{array}$ \\
\hline $\mathrm{Ag}_{4}$ & $\begin{array}{l}\mathbf{1} \mathbf{B}_{1 \mathbf{u}} \\
2 B_{2 u} \\
4 B_{3 u}\end{array}$ & $\begin{array}{l}3.10 \\
4.37 \\
5.68\end{array}$ & $\begin{array}{l}400 \\
284 \\
218\end{array}$ & $\begin{array}{l}\mathbf{0 . 8 4 7} \\
0.269 \\
0.199\end{array}$ & $\begin{array}{l}17 b_{1 u} \rightarrow 24 a_{g}, 95 \% \\
23 a_{g} \rightarrow 17 b_{2 u}, 51 \% \\
23 a_{g} \rightarrow 11 b_{3 u}, 87 \%\end{array}$ \\
\hline $\mathrm{Ag}_{4}^{+}$ & $\begin{array}{l}2 \mathbf{B}_{1 \mathbf{u}} \\
4 B_{2 u}\end{array}$ & $\begin{array}{l}3.22 \\
5.31\end{array}$ & $\begin{array}{l}385 \\
279\end{array}$ & $\begin{array}{l}\mathbf{0 . 5 5 8} \\
0.284\end{array}$ & $\begin{array}{l}17 \alpha b_{1 u} \rightarrow 24 \alpha a_{g}, 62 \% \\
10 \beta b_{3 g} \rightarrow 17 \beta b_{1 u}, 25 \% \\
23 \alpha a_{g} \rightarrow 17 \alpha b_{2 u}, 22 \% \\
23 \beta a_{g} \rightarrow 17 \beta b_{2 u}, 22 \%\end{array}$ \\
\hline $\mathrm{Ag}_{5}$ & $\begin{array}{l}4 \mathbf{B}_{2} \\
5 A_{1} \\
6 B_{1}\end{array}$ & $\begin{array}{l}3.17 \\
3.55 \\
\\
4.79 \\
\end{array}$ & $\begin{array}{l}391 \\
349 \\
\\
259\end{array}$ & $\begin{array}{l}\mathbf{0 . 9 4 7} \\
0.589 \\
0.300\end{array}$ & $\begin{array}{l}38 \alpha b_{2} \rightarrow 46 \alpha a_{1}, 42 \% \\
44 \beta a_{1} \rightarrow 45 \beta a_{1}, 23 \% \\
38 \alpha b_{2} \rightarrow 39 \alpha b_{2}, 22 \% \\
38 \beta b_{2} \rightarrow 39 \alpha b_{2}, 20 \% \\
38 \beta b_{2} \rightarrow 17 \beta a_{2}, 49 \%\end{array}$ \\
\hline $\mathrm{Ag}_{5}^{+}$ & $\begin{array}{l}1 \mathbf{B}_{\mathbf{2}} \\
14 E\end{array}$ & $\begin{array}{l}3.34 \\
4.58\end{array}$ & $\begin{array}{l}371 \\
271\end{array}$ & $\begin{array}{l}\mathbf{0 . 6 9 7} \\
0.484\end{array}$ & $\begin{array}{l}22 b_{2} \rightarrow 24 a_{1}, 93 \% \\
7 b_{1} \rightarrow 29 e, 38 \% \\
22 b_{2} \rightarrow 30 e, 37 \%\end{array}$ \\
\hline
\end{tabular}

Примечание. Переходы с наибольшей интенсивностью выделены жирным шрифтом.

\section{Выводы}

В рамках теории функционала плотности проведено сравнение структурных, энергетических и оптических свойств заряженных и нейтральных молекулярных кластеров серебра $\operatorname{Ag}_{n}^{q}(n=2-5, q=0,+1)$. Электронные переходы нейтральных МК смещены в сторону меньших энергий по сравнению с катионными. Силы осцилляторов нейтральных МК превышают силы осцилляторов заряженных МК. Сопоставление результатов моделирования с полученными ранее экспериментальными результатами для стекол с МК серебра позволило объяснить эффекты, наблюдаемые в эксперименте, и выработать рекомендации для создания люминесцентных стекол с МК серебра для практических применений.

Работа выполнена при государственной финансовой поддержке Российского научного фонда (Соглашение № 14-23-00136).

\section{Список литературы}

[1] Lu Y., Chen W. // Chem. Soc. Rev. 2012. V. 41. P. 3594. doi $10.1039 / \mathrm{c} 2 \mathrm{cs} 15325 \mathrm{~d}$

[2] Zhang L., Wang E. // Nano Today. 2014. V. 9. P. 132. doi 10.1016/j.nantod.2014.02.010

[3] Diez I., Ras R.H.A. // Nanoscale. 2011. V. 3. P. 1963. doi 10.1039/c1nr00006c

[4] Chen L.-Y., Wang C.-W., Yuan Z., Chang H.-T. // Anal. Chem. 2015. V. 87. P. 216. doi $10.1021 /$ ac503636j

[5] Kuznetsov A.S., Tikhomirov V.K., Shestakov M.V., Moshchalkov V.V. // Nanoscale. 2013. V. 5. P. 10065. doi $10.1039 / \mathrm{c} 3 \mathrm{nr} 02798 \mathrm{~h}$

[6] Ho J., Ervin K.M., Lineberger W.C. // J. Chem. Phys. 1990. V. 93. P. 6987. doi $10.1063 / 1.459475$

[7] Kellerman R. // J. Chem. Phys. 1979. V. 70. P. 1562. doi $10.1063 / 1.437550$

[8] Lecoultre S., Rydlo A., Buttet J., Felix C., Gilb S., Harbich W. // J. Chem. Phys. 2011. V. 134. P. 184504. doi $10.1063 / 1.3589357$ 
[9] Ozin D.A., Hubert H. // Inorg. Chem. 1978. V. 17. P. 155. doi 10.1021/ic50188a031

[10] Wang Y., Gong X.G. // Eur. Phys. J. D. 2005. V. 34. P. 19. doi 10.1140/epjd/e2005-00103-0

[11] Gamboa G.U., Reber A.C., Khanna S.N. // New J. Chem. 2013. V. 37. P. 3928. doi $10.1039 / \mathrm{c} 3 n j 01075 \mathrm{a}$

[12] Fournier R. // J. Chem. Phys. 2001. V. 115. P. 2165. doi $10.1063 / 1.1383288$

[13] Yabana K., Bertsch G.F. // Phys. Rev. A. 1999. V. 60. P. 3809. doi 10.1103/physreva.60.3809

[14] Zhao G.F., Lei Y., Zeng Z. // Chem. Phys. 2006. V. 327. P. 261. doi 10.1016/j.chemphys.2006.04.014

[15] Bonačić -Koutecký V., Pittner J., Boiron M., Fantucci P. // J. Chem. Phys. 1999. V. 110. P. 3876. doi 10.1007/978-3-642-88188-6_36

[16] Игнатьев А.И., Никоноров Н.В., Сидоров А.И., Шахвердов T.A. // Опт. и спектр. 2013. Т. 114. C. 838; Ignatév A.I., Nikonorov N.V., Sidorov A.I., Shakhverdov T.A. // Opt. Spectrosc. 2013. V. 114. P. 769. doi $10.1134 / \mathrm{s} 0030400 \times 13030132$

[17] Dubrovin V.D., Ignatiev A.I., Nikonorov N.V., Sidorov A.I., Shakhverdov T.A., Agafonova D.S. // Opt. Mater. 2014. V. 36. P. 753. doi 10.1016/j.optmat.2013.11.018

[18] ADF2014, Vrije Universiteit, Amsterdam, Netherlands. 2014. http://www.scm.com.

[19] Perdew P., Burke K., Ernzerhof M. // Phys. Rev. Lett. 1996. V. 77. P. 3865. doi 10.1103/physrevlett.77.3865

[20] Gross E.K.U., Dobson J.F., Petersilka M. in Topics in Current Chemistry. Springer, 1996. V. 181. P. 81. doi $10.1007 / \mathrm{bfb} 0016643$

[21] Rabilloud F. // J. Phys. Chem. A. 2013. V. 117. P. 4267. doi $10.1021 /$ jp3124154

[22] Yanai T., Tew D.P., Handy N.C. // Chem. Phys. Lett. 2004. V. 393. P. 51. doi 10.1016/j.cplett.2004.06.011

[23] Simard B., Hackett P.A., James A.M., LangridgeSmith P.R.R. // Chem. Phys. Lett. 1991. V. 186. P. 415. doi 10.1016/0009-2614(91)90201-j

[24] Boo D.W., Ozaki Y., Andersen L.H., Lineberger W.C. // J. Phys. Chem. A. 1997. V. 101. P. 6688. doi 10.1021/jp9711353

[25] Kittel C. Introduction to Solid State Physics. Wiley, 2004. $704 \mathrm{p}$. 